\title{
Analysis of magnetic resonance imaging-based blood and cerebrospinal fluid flow measurements in patients with Chiari I malformation: a system approach
}

\author{
Noam Alperin, Ph.D., Kirti Kulkarni, M.D., Ben Roitberg, M.D., \\ Francis Loth, Ph.D., Naren K. Pandian, Mahmood F. Mafee, M.D., \\ Mina Foroohar, M.D., ANd Terry Lichtor, M.D., Ph.D. \\ Departments of Radiology, Neurosurgery, and Mechanical Engineering, University of Illinois at \\ Chicago; and Department of Neurological Surgery, Rush Medical College and Cook County Hospital, \\ Chicago, Illinois
}

\begin{abstract}
Object. A pilot study was performed to assess noninvasively the change in intracranial compliance (ICC) and intracranial pressure (ICP) in patients with Chiari I malformation who undergo foramen magnum decompression. The working hypothesis was that the main effect of the decompressive surgery is a change in ICP. Noninvasive cine phasecontrast magnetic resonance (MR) imaging is a motion-sensitive dynamic MR imaging technique that allows for visualization and quantitation of tissue motion and flow. The authors' group has used dynamic phase-contrast MR imaging to visualize and quantify pulsatile blood and cerebrospinal fluid (CSF) flow in the craniospinal system.

Methods. A system approach has been used to characterize the hemodynamic-hydrodynamic coupling in the craniospinal system and to derive measures for ICC and ICP. Magnetic resonance imaging-based ICC and ICP values are derived from the ratio of the volume and pressure changes that occur naturally during each cardiac cycle. The authors conducted a prospective study of four patients, three of whom were studied before and after decompressive surgery; significant change in MR imaging-derived ICC and ICP values was documented in only one of the three surgically treated patients. A significant change in the dynamics of the intracranial volume change (ICVC) during the cardiac cycle, however, was observed in all three patients. In healthy individuals the ICVC waveform usually consists of the following sequence: monotonic increase in intracranial volume (ICV) during the systolic phase due to increased blood inflow, monotonic decrease in ICV caused by the onset of CSF outflow into the spinal canal, and increase in the venous outflow. A nonmonotonic decline in the ICVC waveform has been observed in all patients with headaches, and a relatively normal waveform was found in those without headaches or whose headaches were resolved or alleviated by the surgery. A "partial-valve" mechanism is proposed as an explanation for the abnormal ICVC dynamics. The monotonic decline in ICVC is interrupted by a "premature" reduction in the CSF outflow. This may be caused by a displacement of the hindbrain into the cervical spinal canal during the systolic phase. This obstructs the CSF flow at the later part of the systolic phase such that the ICV does not continue its gradual decline. Postsurgery, the ICVC waveforms presented a more normal-appearing ICVC dynamics profile.

Conclusions. Magnetic resonance imaging measurement of transcranial CSF and blood flow may lead to a better understanding of the pathophysiology of Chiari malformations and may prove to be an important diagnostic tool for guiding for the treatment of patients with Chiari I malformation.
\end{abstract}

\section{KEY WORDS - Arnold-Chiari malformation - cerebrospinal fluid dynamics • transcranial blood flow • magnetic resonance imaging • intracranial compliance • cine phase-contrast intracranial volume change}

Magnetic resonance imaging studies obtained in patients with Chiari I malformations have traditionally been confined to anatomical images. Further insight into the pathophysiology of the Chiari I malformation has been acquired using phase-contrast MR imaging of the cervical CSF and spinal cord. ${ }^{4,8,9,15}$ This modality allows visualiza-

Abbreviations used in this paper: CSF = cerebrospinal fluid; ICA = internal carotid artery; ICC = intracranial compliance; ICP = intracranial pressure; ICV = intracranial volume; ICVC $=\mathrm{ICV}$ change; $\mathrm{MR}=$ magnetic resonance; $\mathrm{VA}=$ vertebral artery . tion and quantification of CSF flow through the foramen magnum region. Reduced CSF flow during systole and diastole has been documented in patients with a Chiari I malformation. ${ }^{8}$ After decompression of the foramen magnum in these patients, the CSF flow through this region increases while the peak CSF pulse pressure decreases. In patients with Chiari I malformations and syringomyelia, this change in CSF flow across the foramen magnum correlates with a decrease in size of the syrinx. ${ }^{8}$

Phase-contrast MR imaging studies were used to elucidate the progress of syringomyelia associated with Chiari 


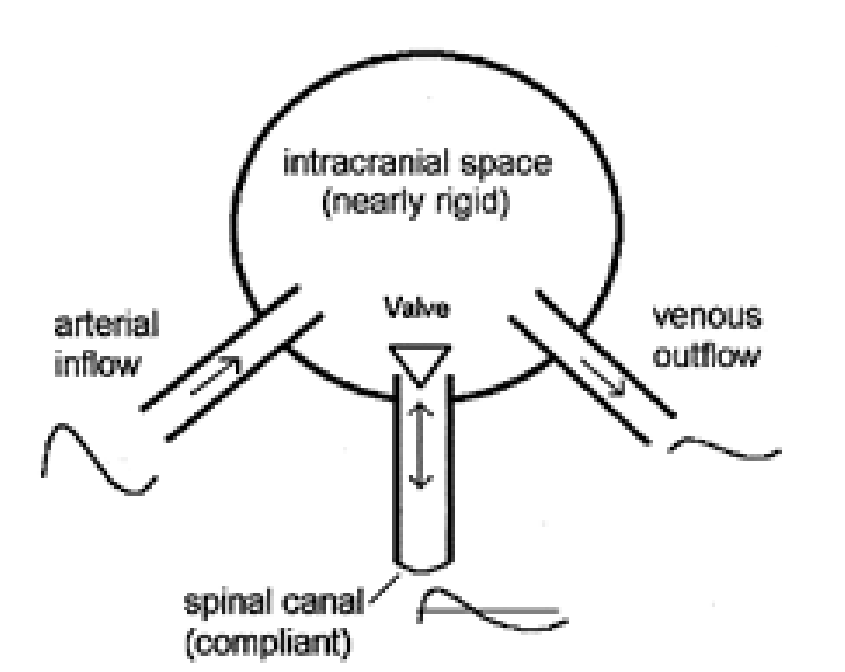

Fig. 1. Compartmental model of the craniospinal system includes the nearly rigid cranium, the compliant spinal canal, and the channels of arterial, venous, and CSF flow.

I malformations by the action of the cerebellar tonsils, which partially occlude the subarachnoid space at the foramen magnum and act as a piston on the enclosed spinal subarachnoid space. ${ }^{8}$ This creates a pressure wave that compresses the spinal cord and propagates CSF within the syrinx caudally, with each heartbeat leading to syrinx progression. Therefore, a decompressive suboccipital craniectomy, a C-1 laminectomy, and duraplasty eliminate this mechanism, resulting in resolution of syringomyelia in most cases. ${ }^{4,8,13,15}$ It has also been suggested that a limited occipital craniectomy, C-1 laminectomy, and dural opening without subsequent duraplasty is a safe and effective treatment. ${ }^{12}$ Some authors have reported that a subset of patients with Chiari I malformation and syringomyelia can be treated with the removal of bone without the need for dural opening. ${ }^{13}$ In contrast, other authors recommend either foramen magnum decompression and placement of a syringosubarachnoid shunt ${ }^{7}$ or placement of a syringosubarachnoid shunt alone ${ }^{10}$ as the optimal surgical treatment for this patient population. The clinical decision and the understanding of this etiology are further complicated by reports of instances of spontaneous resolution of the syrinx. ${ }^{5,11}$

The role of increased ICP is not well established in patients with Chiari malformations. Our research group has developed an MR imaging-based method for measurement of ICC and ICP. ${ }^{3}$ Intracranial compliance and ICP may be important for characterization of the intracranial hydrodynamics in patients with a variety of neurological problems including Chiari I malformations. The MR imaging technique utilizes the pulsatile changes in the ICV and ICP that occur naturally during the cardiac cycle. The elastance (the inverse of compliance) is estimated by the ratio of these changes and is linearly related to absolute CSF pressure through the elastance curve. ${ }^{3}$ The total ICVC during the cardiac cycle is derived from net transcranial blood and CSF volumetric flow rates. The pressure change is estimated from the pulsatile CSF pressure gradient waveform that is calculated from the CSF velocities determined by phase-contrast MR imaging. ${ }^{3}$

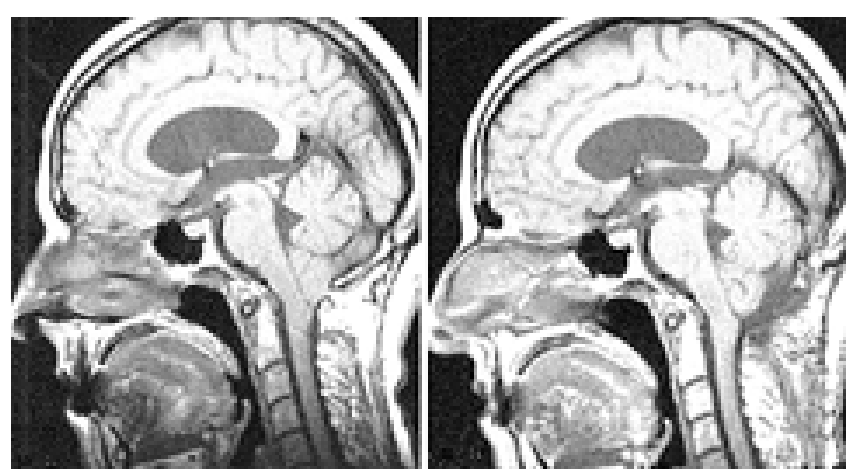

Fig. 2. Case 1. Predecompression (left) and postdecompression (right) midsagittal spin echo $\mathrm{T}_{1}$-weighted MR images. Ventricles remained enlarged after the decompression.

In this report, a system approach is applied in the analysis of the MR imaging studies obtained in patients with Chiari I malformation. In this approach, the craniospinal system is represented by a nearly rigid cranial compartment connected to a more compliant spinal canal through a CSF flow channel. ${ }^{2}$ The CSF flow between the cranium and the spinal canal is dynamically modulated by a partial obstruction (partial valve) at the level of the foramen magnum. Cerebrospinal fluid pulsation originates from pulsatile blood flow and is modulated by the mechanical compliance of the craniospinal system. ${ }^{2}$ The net arterial inflow into the cranium is the sum of arterial inflow through the four main arteries, the left and right ICAs and VAs. The net venous outflow is the venous flow through the jugular veins and, when present, a secondary flow channel through the epidural veins. The proposed compartmental model of the craniospinal system is shown in Fig. 1.

TABLE 1

Summary of MR imaging-derived hemo- and hydrodynamic parameters*

\begin{tabular}{cccccc}
\hline \hline $\begin{array}{c}\text { Case } \\
\text { No. }\end{array}$ & $\begin{array}{c}\text { CSF Osc } \\
\text { Vol }^{\dagger}(\mathrm{ml})\end{array}$ & $\begin{array}{c}\text { Pulsatility } \\
\text { Ratio }^{\dagger}\end{array}$ & $\begin{array}{c}\text { ICVC } \$ \\
(\mathrm{ml})\end{array}$ & $\begin{array}{c}\text { ICSVCll } \\
(\mathrm{ml})\end{array}$ & $\begin{array}{c}\text { ICP } \\
(\mathrm{mm} \mathrm{Hg})\end{array}$ \\
\hline $1^{* *}$ & & & & & \\
$\quad$ preop & 0.49 & 0.44 & 0.34 & 0.84 & 13 \\
$\quad$ postop & 0.50 & 0.45 & 0.32 & 1.06 & 13 \\
$2^{\dagger \dagger}$ & & & & & \\
$\quad$ preop & 0.70 & 0.61 & 0.50 & 0.46 & 7 \\
postop & 0.88 & 0.52 & 0.45 & 0.86 & 11 \\
$3 \begin{array}{l}\text { preop } \\
4\end{array}$ & 0.60 & 1.06 & 1.36 & 1.18 & 6 \\
preop & 0.27 & 0.78 & 0.16 & 0.32 & 19 \\
postop & 0.43 & 0.38 & 0.56 & 0.90 & 6 \\
\hline
\end{tabular}

* ICSVC = intracraniospinal volume change; Osc = oscillatory; $\mathrm{Vol}=$ volume.

$\dagger$ Volume of CSF that flows in each cardiac cycle between the cranium and spinal canal.

$\ddagger$ Pulsatility ratio is defined as the ratio between peak-to-peak amplitudes of the venous outflow and the arterial inflow.

$\S$ Intracranial volume change during each cardiac cycle.

|| Intracraniospinal volume change during each cardiac cycle.

** The patient also harbored a syrinx.

$\dagger \dagger$ The patient also had hydrocephalus. 

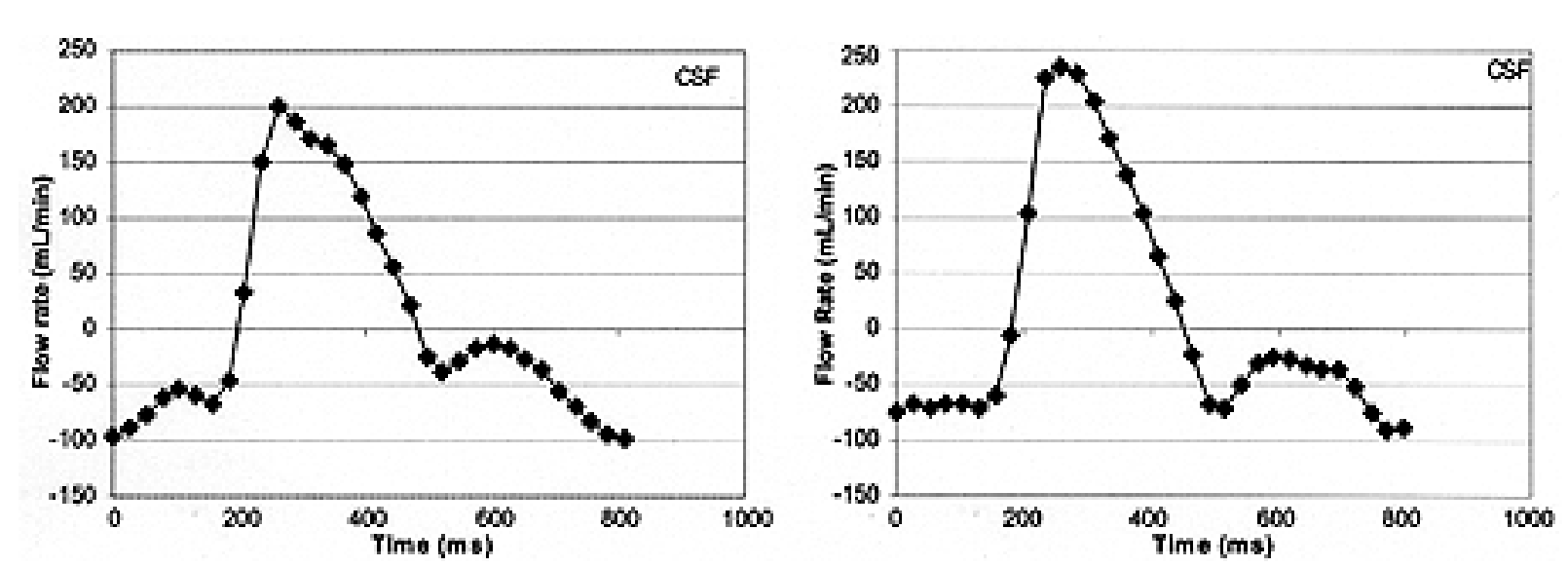

Fig. 3. Case 1. Preoperative (left) and postoperative (right) CSF waveforms. No significant change was observed in the duration of the CSF outflow during systolic phase as well as in oscillatory CSF volume (see Table 1). The peak flow rate, however, increased postoperatively $. \min =$ minute $; \mathrm{ms}=$ millisecond.

The goal of this preliminary investigation is to apply a system approach analysis to phase-contrast MR imaging studies obtained in patients with Chiari I malformation before and after undergoing decompressive surgery. We present a correlation between our initial findings and the patients' symptoms and suggest a possible connection between the two.

\section{CLINICAL MATERIAL AND METHODS}

\section{Patient Population}

We performed a prospective study of four patients in whom Chiari I malformation was diagnosed based on tonsillar herniation below the foramen magnum as demonstrated on sagittal T-weighted MR images. Each patient's imaging studies included sagittal and axial $\mathrm{T}_{1}-$ and $\mathrm{T}_{2}-$ weighted MR images as well as two sets of cine phasecontrast MR images for quantitation of blood and CSF flow at the level of C-2. ${ }^{1-2}$ In one patient, a midsagittal cine phase-contrast MR study scan was also obtained to measure the hindbrain displacement during the cardiac cycle. Three of the four patients were studied before and after they underwent a decompressive procedure.

\section{Phase-Contrast MR Imaging Analysis}

Imaging Protocol. Magnetic resonance images were obtained using a velocity-encoded cine phase-contrast pulse sequence with peripheral gating. Transcranial blood (arterial and venous) and CSF volumetric flow rates were measured according to a technique previously described. ${ }^{2,3}$ Two separate images in a transverse or oblique section just below the foramen magnum were obtained. The first image was used to measure CSF and spinal cord oscillatory pulsation, and the second image was used to measure arterial inflow through the ICAs and VAs and venous outflow through the jugular veins. In each case, a 4- to 6-mm-thick section was imaged with a 12 - to $16-\mathrm{cm}$ field of view, a $256 \times 128$ or $256 \times 160$ matrix, and an average of two excitations were acquired. Velocity was encoded along the superior-to-inferior axis with a gradient strength chosen for velocities of interest to be just below the aliasing velocity (velocity corresponding to $180^{\circ}$

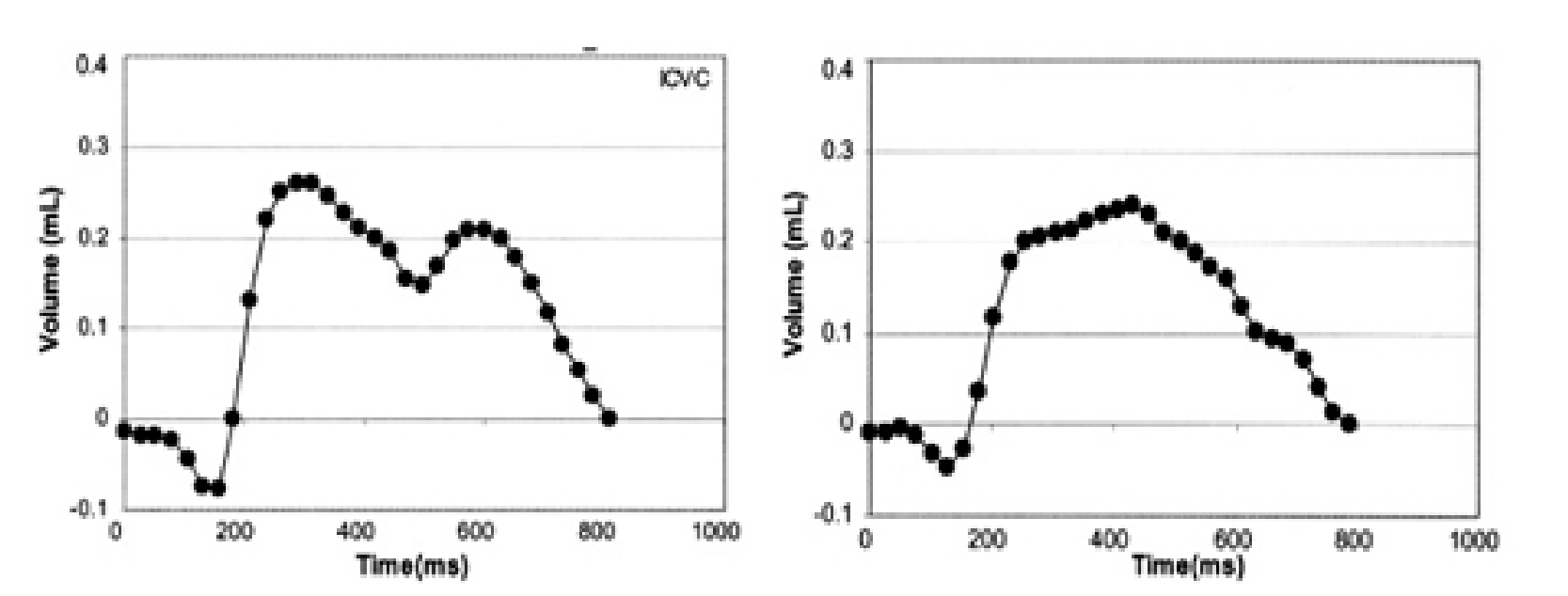

Fig. 4. Case 1. Preoperative and postoperative ICVC waveforms. Left: The preoperative waveform has two distinct peaks. Right: The postoperative waveform has only one peak. The total ICVC is similar (Table 1). 


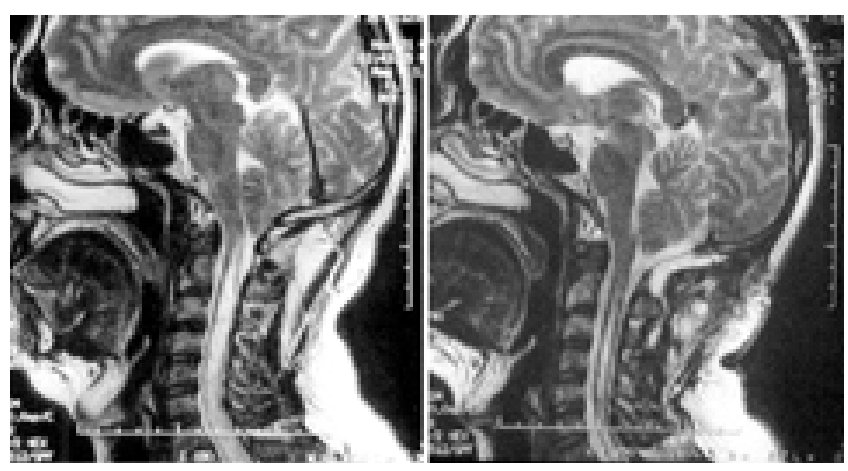

Fig. 5. Case 2. Predecompression (left) and postdecompression (right) midsagittal $\mathrm{T}_{2}$-weighted MR images. Note the marked decrease in size of the syrinx after the foramen magnum was decompressed.

phase value). Cerebrospinal fluid flow was measured with a velocity-encoding value of 5 to $9 \mathrm{~cm} / \mathrm{second}$, and blood flow was measured with a velocity-encoding value of 60 to $90 \mathrm{~cm} / \mathrm{second}$. The shortest available repetition time, 17 to $22 \mathrm{msec}$, was used to optimize spatial resolution and to minimize low-pass filtering of the temporal waveform caused by data interpolation. The echo time and flip angle were 6.5 to $9 \mathrm{msec}$ and 20 to $25^{\circ}$, respectively. The data were interpolated to the maximum number of time points available per cardiac cycle (32) to minimize errors due to secondary resampling.

Magnetic Resonance Imaging-Derived and Craniospinal Volume Change. Time-varying ICVC was computed from the net transcranial volumetric flow of blood and CSF. The transcranial volumetric blood flow rate was measured in each of the following six blood vessels: bilateral ICAs, VAs, and internal jugular veins. A region of interest was drawn by one of the authors (K.K.) around each vessel lumen. The mean volumetric flow rate was calculated by multiplying the mean velocity by the lumen area.

Because brain tissue, blood, and CSF are all incompressible, the volume change can be calculated directly from the net volumetric flow rates into and out of the cranium, as described by these two equations:

$\operatorname{ICVC}(\mathrm{t})=\left[\mathrm{Q}_{\mathrm{A}}(\mathrm{t})-\mathrm{Q}_{\mathrm{V}}(\mathrm{t})-\mathrm{Q}_{\mathrm{CSF}}(\mathrm{t})\right] \Delta \mathrm{t}$

and

$\sum_{\mathrm{T}}^{\Sigma} \operatorname{ICVC}(\mathrm{t})=\Sigma\left[\mathrm{Q}_{\mathrm{A}}(\mathrm{t})-\mathrm{Q}_{\mathrm{V}}(\mathrm{t})-\mathrm{Q}_{\mathrm{CSF}}(\mathrm{t})\right] \Delta \mathrm{t}=0$

where ICVC is the intracranial volume change, $Q_{A}(t)$ is the total arterial volumetric flow rate, $Q_{y}(t)$ is the total venous volumetric outflow rate, and $Q_{C S F}(t)$ is the rate of CSF volumetric outflow through the foramen magnum. The $\mathrm{t}$ is a time point within the cardiac cycle, $\Delta \mathrm{t}$ is the interval between two time points, and $\mathrm{T}$ is the time period of one cardiac cycle. The second equation represents the Monro doctrine, which states that the total ICV is constant. Because ICV fluctuates during the cardiac cycle, however, the Monro doctrine holds only for the mean volume (mean ICV is constant). The second equation is used to remove bias in the MR imaging-detected velocity measurements. ${ }^{1}$ The total ICVC is derived by integration of the rate of transcranial volumetric flow rate and finding the difference between the maximum and minimum of the integral. In addition to the change in ICV, the total change in the volume of the intracraniospinal system was similarly derived from the difference between the arterial inflow and venous outflow excluding the flow within the craniospinal system. The intracraniospinal volume change (ICSVC) is defined as follows:

$\operatorname{ICSV}(\mathrm{t})=\left[\mathrm{Q}_{\mathrm{A}}(\mathrm{t})-\mathrm{Q}_{\mathrm{V}}(\mathrm{t})\right] \Delta \mathrm{t}$.

Magnetic Resonance Imaging-Derived Hemo- and Hydrodynamics Parameters. In addition to the preceding parameters, the oscillatory CSF volume and the pulsatility ratio were also derived from the MR imaging flow study. The oscillatory CSF volume is the volume of CSF that flows between the cranium and the spinal canal in each cardiac cycle. ${ }^{2}$ The pulsatility ratio was calculated from the measured arterial and venous volumetric flow rate waveforms. It is defined as the ratio between the peak-to-peak amplitudes of the venous outflow and the arterial inflow.

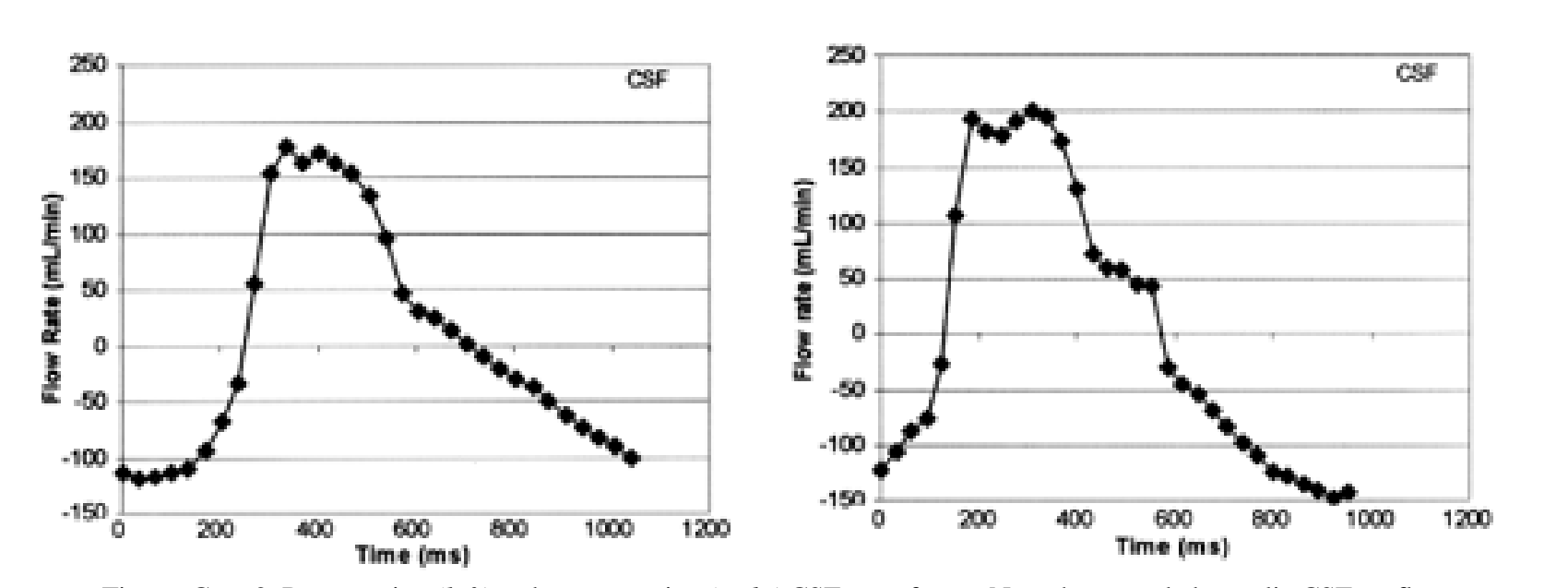

Fig. 6. Case 2. Preoperative (left) and postoperative (right) CSF waveforms. Note the extended systolic CSF outflow after decompression. The peak flow slightly increased after surgery. 

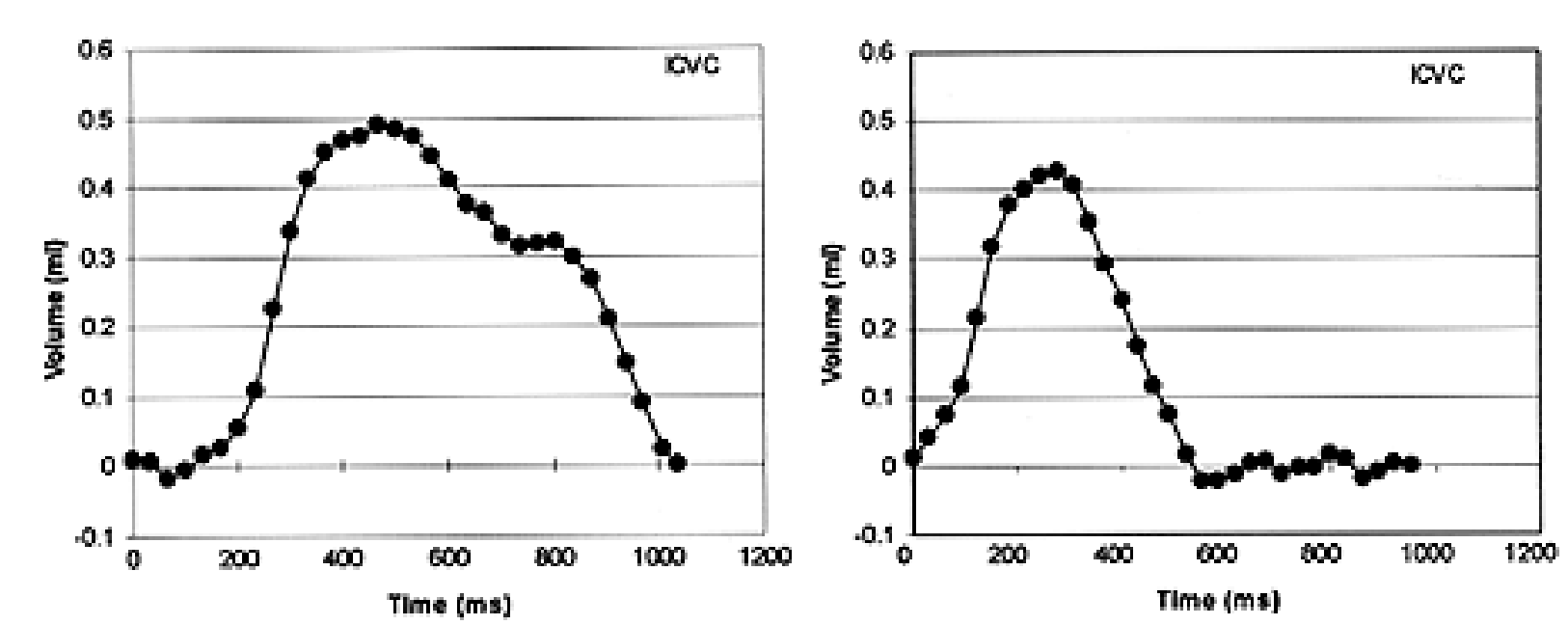

Fig. 7. Case 2. Preoperative (left) and postoperative (right) ICVC waveforms. A shorter duration of the increased volume was measured postdecompression.

Preoperative and postoperative hindbrain displacement measurements were obtained for the last cases by performing midsagittal cine phase-contrast MR imaging with velocity encoding of $7 \mathrm{~cm} /$ second in the superoinferior direction. The displacement in millimeters was obtained from an integration of the mean velocity in the hindbrain region.

\section{RESULTS}

The quantitative hemo- and hydrodynamic parameters are summarized in Table 1. The clinical background, observations, and the quantitative results obtained in each patient are described.

\section{Case 1: Chiari I Malformation With Hydrocephalus}

This 41-year-old right-handed woman presented with a long-standing history of headaches. The only significant finding on her workup was an electroencephalogram-detected abnormal focus in the left temporal region. The patient was treated with Dilantin. Preoperative MR imag-

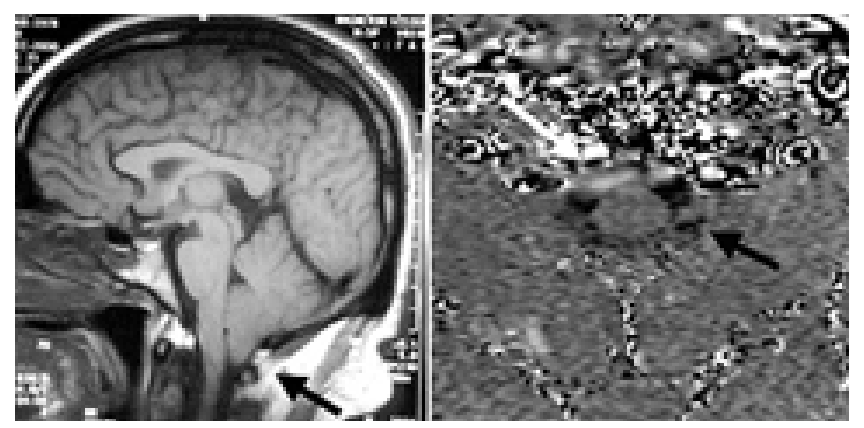

Fig. 8. Case 3. Left: Midsagittal $\mathrm{T}_{1}$-weighted MR image. Note the caudal descent of the cerebellar tonsils $10 \mathrm{~mm}$ from the tip of the foramen magnum. Right: Phase-contrast MR image demonstrating irregular CSF velocities in opposite directions within the cervicospinal subarachnoid space. Arrows indicate flow moving in opposite directions (black arrow: caudocranial; white arrow: craniocaudal at the same cross section). ing revealed an 8-mm tonsillar herniation below the level of the foramen magnum that extended to the posterior arch of $\mathrm{C}-1$. The studies also revealed hydrocephalus with moderate dilation of the lateral and third ventricles and mild dilation of the fourth ventricle. The patient underwent a suboccipital craniectomy, C-1 laminectomy, and duraplasty. A postoperative MR imaging study was obtained 6 weeks after surgery.

Postoperatively, this patient experienced a dramatic decrease in the severity of her headaches. Her pre- and postdecompression midsagittal spin echo $\mathrm{T}_{1}$-weighted $\mathrm{MR}$ images are shown in Fig. 2. The postoperative image demonstrated that the ventricles remained enlarged. The preand postoperative CSF waveforms are shown in Fig. 3. The calculated oscillatory CSF volume was unchanged, and no significant change in duration of CSF outflow during systole was noticed. The peak CSF flow, however, was higher after surgery (240 [postoperatively] compared with $200 \mathrm{ml} /$ minute [preoperatively]). The MR imaging-derived ICC and ICP measurements were found to be moderately above normal values both pre- and postoperatively (Table 1). The pre- and postoperative ICVC waveforms are shown in Fig. 4. There was no statistically significant alteration in total ICVC (Table 1), but the shape of the integrated ICVC waveforms changed significantly. The preoperative ICVC waveform showed two peaks with lower amplitude whereas only one peak, which is a more normal shape, was observed in the postoperative study.

\section{Case 2: Chiari I Malformation With Syrinx}

This 35-year-old right-handed man presented with a 1-year history of right-hand weakness and dysesthesia in both hands associated with neck pain and no headaches. Positive findings on neurological examination included decreased strength $(4+/ 5)$ in the right hand and a diminished pinprick sensitivity over the medial aspect of both forearms. A preoperative MR image revealed 10-mm tonsillar herniation below the foramen magnum as well as a large syrinx extending from C-2 to T-7. The patient underwent a suboccipital craniectomy, C-1 laminectomy, and 

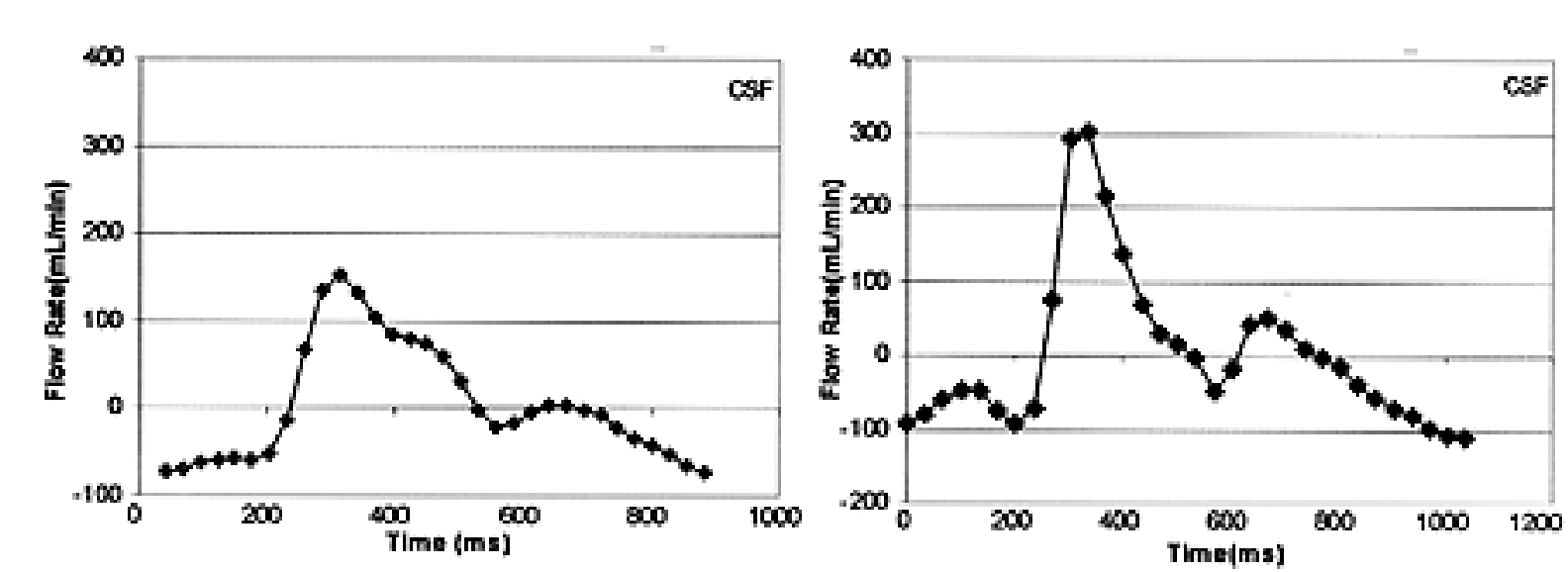

Fig. 9. Cerebrospinal fluid flow waveforms obtained in a healthy volunteer (left) and in the patient in Case 3. The patient's CSF waveform is pulsatile compared with that obtained in the healthy individual.

duraplasty. A postoperative MR image was obtained 8 weeks after surgery.

Postoperatively the patient's dysesthesia diminished, and the symptoms in his left arm completely resolved. He also regained strength in his right arm, although he still experienced moderate weakness of grip strength 6 months postoperatively. Preoperative and postoperative sagittal $\mathrm{T}_{2}$-weighted MR images are shown in Fig. 5. Postoperative MR imaging revealed a decrease in the size of the syrinx. Preoperative and postoperative CSF flow waveforms are shown in Fig. 6. The postoperative CSF waveform showed a longer period of CSF outflow during systole when compared with the preoperative CSF waveform. The oscillatory CSF volume increased from 0.70 to 0.88 $\mathrm{ml}$ (Table 1). The pre- and postoperative IVC waveforms are shown in Fig. 7. Total ICVC decreased from 0.5 to $0.45 \mathrm{ml}$ (Table 1), but this change is statistically insignificant because it is within the measurement error of $18 \% .^{3}$ The shape of the waveform, however, changed. A shorter duration of increased volume was noted on the postoperative waveform. Calculated MR imaging-derived ICC and ICP values were slightly higher postoperatively but with- in normal range both before and after decompressive surgery (Table 1).

\section{Case 3}

This 22-year-old right-handed man presented with a 1.5-year history of headaches. Magnetic resonance imaging revealed 9-mm tonsillar herniation but no evidence of hydrocephalus or a syrinx. Three months later, neuroimaging studies that included phase-contrast MR imaging were performed. Again the images demonstrated significant tonsillar herniation. At that time, however, the headaches had resolved and there was no need to perform decompressive surgery.

The sagittal $\mathrm{T}_{1}$-weighted image shown in Fig. 8. left demonstrates the caudal descent of the cerebellar tonsils $10 \mathrm{~mm}$ below the tip of the foramen magnum. The patient's symptoms resolved without surgery. He was symptom free for 2 months prior to undergoing the phase-contrast MR imaging study. An axial cine phase-contrast image obtained during the end systolic phase is shown in Fig. 8 right. The velocity image reveals uneven distribution of CSF flow and, at the same instance, flow both in
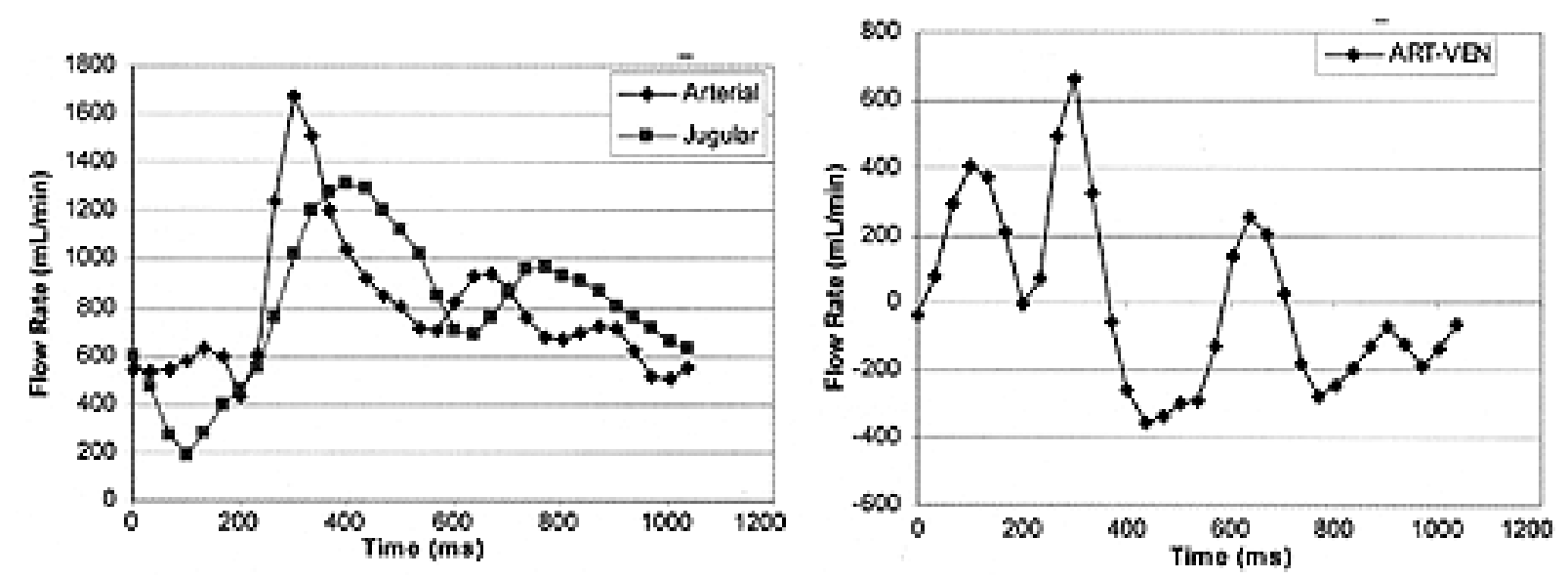

Fig. 10. Case 3. Arterial and venous flow waveforms (left), which demonstrate a highly pulsatile jugular flow and the net transcranial flow (right), which is the arterial inflow minus the venous outflow (ART-VEN). 


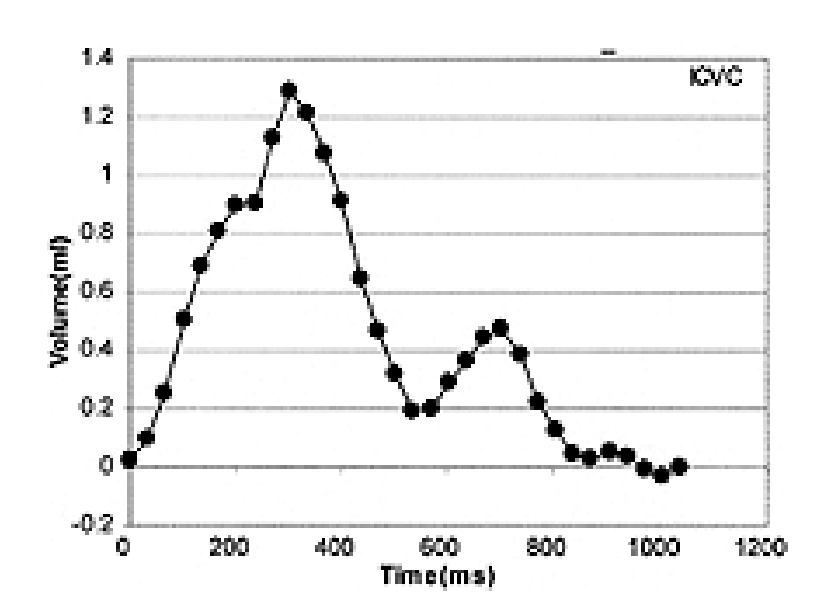

Fig. 11. Case 3. The ICVC waveform shows a large peak and a small secondary peak. This patient was not symptomatic for headaches at the time of the MR imaging study.

superoinferior and inferosuperior directions, at the anterior part of the cervical subarachnoid space. Examples of CSF flow waveforms obtained in a healthy volunteer and in this patient are shown in Fig. 9. The oscillatory CSF volume in this patient was within the normal range of 0.7 $\pm 0.2 \mathrm{ml}$ reported in our earlier study $;^{2}$ however, the overall CSF flow was highly pulsatile.

The total arterial and jugular flow waveforms are demonstrated in Fig. 10 left. The jugular waveform was unusually pulsatile. In particular the jugular flow revealed pulse amplitude similar to that of the arterial flow (pulsatility ratio of 1). This results in an anomaly of a negative blood flow (net outflow of blood from the cranium) during systole, as can be seen in the arterial minus venous flow waveforms shown in Fig. 10 right. The ICVC waveform, shown in Fig. 11, demonstrates a large peak with a small secondary peak. The ICP measured in this patient was $6 \mathrm{~mm} \mathrm{Hg}$, which is well within the normal range of values. ${ }^{3}$

A video animation of the pulsatile blood and CSF volumentric flow during the cardiac cycle provides a visual tool to understand more easily the dynamics of the intracranial volume change. The abnormal hemodynamics and the resultant ICVC dynamic of the patient described in Case 3 are demonstrated in the video animation.

CLICK HERE TO VIEW VIDEO ANIMATION. This animation illustrates the volumetric flow rates into and from the cranium and the resultant intracranial volume change during the cardiac cycle. The volumetric arterial inflow is shown in red, the venous outflow in blue, and the CSF oscillatory flow in yellow. The change in the total intracranial volume is shown in orange. The corresponding waveforms are seen on the top and bottom left: the ICVC waveforms on the top and the blood and CSF volumetric flow rate waveforms on the bottom. This animated video demonstrates the coupling of the hemodynamics and hydrodynamics of the craniospinal system.

\section{Case 4}

This 37-year-old right-handed woman presented with a history of left shoulder pain and 1.5-year history of mild winging of the scapula. Her history was significant for multiple left-ear eustachian tube problems. She developed numbness and intermittent pain in the left C-7 distribution. Throughout this period, the patient complained of severe (10 on a scale of 10), mainly occipital, headaches. The patient underwent a suboccipital craniectomy, C-1 laminectomy, and duraplasty. A postoperative MR imaging study was performed 3 weeks after surgery. Postoperatively the patient did not take any medication for headaches, which subsided in severity (seven on a scale of 10).

Sagittal pre- and postoperative $\mathrm{T}_{1}$-weighted images are shown in Fig. 12 left and center. The caudal descent of the cerebellar tonsils presurgery was approximately $10 \mathrm{~mm}$. Hemodynamic factors changed significantly after the surgery. The preoperative pulsatility ratio was relatively high before the surgery (approximately 0.78 ) and was significantly reduced $(0.38)$ postsurgery. The patient's pre- and postsurgery rest heart rates measured during the MR imaging study were $78 \mathrm{bpm}$ and $57 \mathrm{bpm}$, respectively. Figure 12 right shows the velocity phase-contrast MR image obtained at the level of C-2. A significant venous outflow in

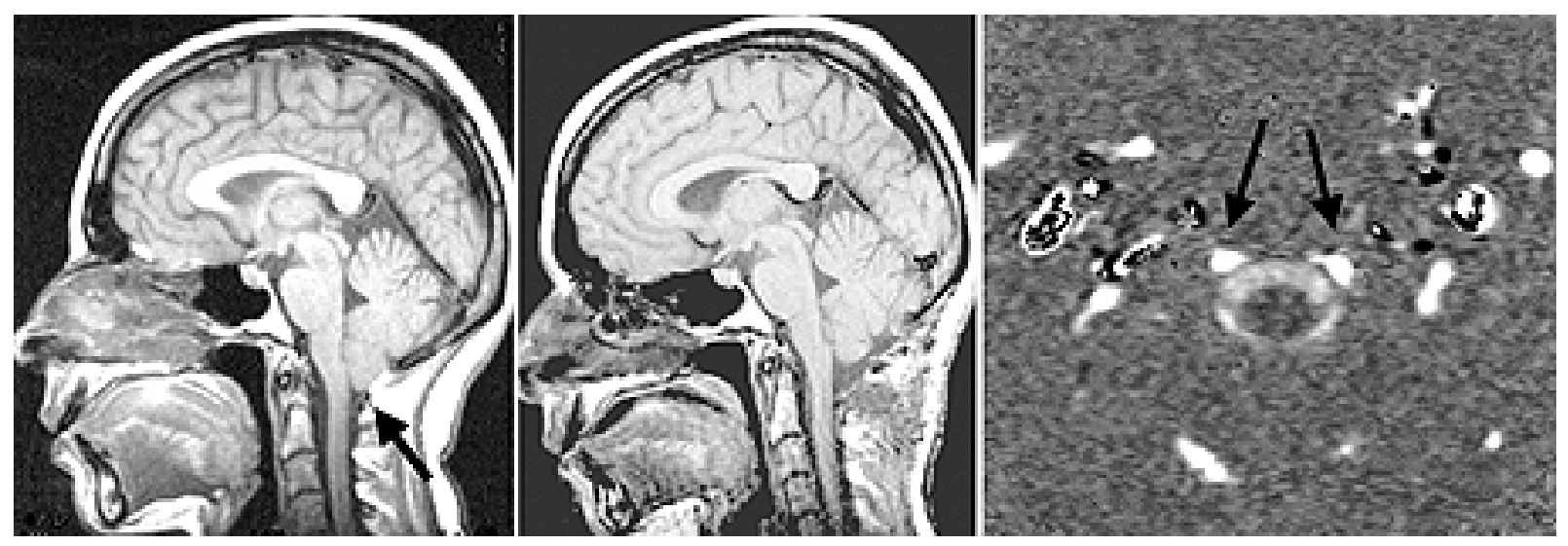

Fig. 12. Case 4. Presurgical (left) and postsurgical (center) spin echo $\mathrm{T}_{1}$-weighted sagittal MR images. Note the caudal descent of the crerebellar tonsils below the tip of the foramen magnum. Right: One of 32 velocity-encoded phasecontrast MR images acquired at mid-C-2 level before decompressive surgery. A significant venous outflow through the epidural veins (black arrows) is observed. Total mean epidural venous outflow was $25 \mathrm{ml} / \mathrm{minute}$. 


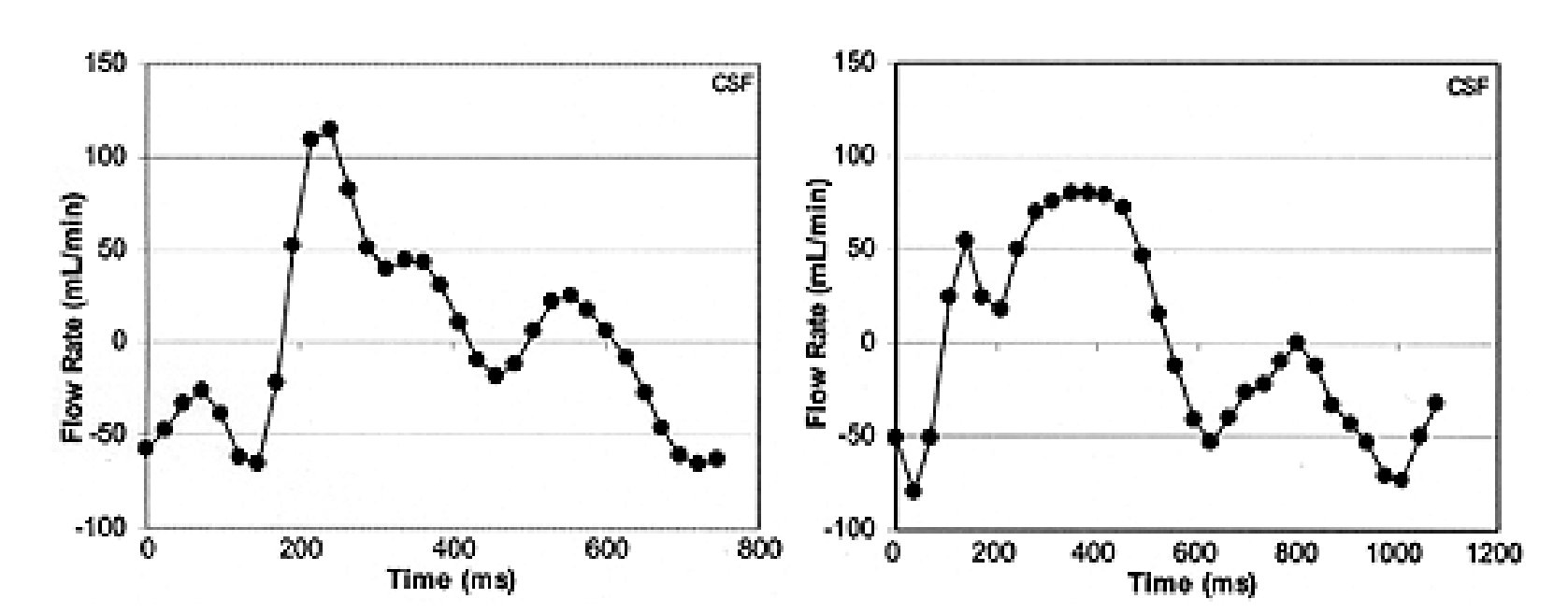

Fig. 13. Case 4. Preoperative (left) and postoperative (right) CSF waveforms. Note that the postoperative peak flow rate is actually lower, but the duration of the superoinferior flow is significantly longer. The oscillatory CSF volume increased significantly after decompressive surgery from 0.27 to $0.43 \mathrm{ml}$.

the epidural veins within the anterior part of the spinal canal was observed. Total pre- and postoperative mean venous outflows through the epidural veins were $25 \mathrm{ml} /$ minute and $16 \mathrm{ml} /$ minute, respectively.

The pre- and postsurgery CSF flow waveforms are shown in Fig. 13. The oscillatory CSF volume increased significantly, from $0.27 \mathrm{ml}$ to $0.43 \mathrm{ml}$. The preoperative peak CSF flow rate was $120 \mathrm{ml} /$ minute, which is relatively low; the postoperative peak flow rate was even lower ( $80 \mathrm{ml} /$ minute), although in the postoperative study, the overall duration of the outflow increased significantly.

The ICVC waveforms are shown in Fig. 14. The total ICVC was altered significantly after the surgery from 0.16 $\mathrm{ml}$ to $0.56 \mathrm{ml}$; this is a statistically significant change. Furthermore, the shape of the ICVC waveform was completely modified after surgery through the altered blood and CSF flow dynamics. Preoperatively there were two distinct low amplitude peaks whereas the postoperative waveform has a more normal-appearing shape with almost monotonic decline. As in previous cases, the "double-peak" shape of the ICVC waveform was associated with symptoms of severe headaches. This patient's MR imaging-derived ICC/ICP has changed from moderately elevated to the low range of normal values (Table 1).

\section{DISCUSSION}

The results of this pilot study demonstrate the wide range of variability in the hemo- and hydrodynamics associated with Chiari I malformation. This may be in part because of the wide variability of pathological entities presented by the four patients. These include Chiari malformation with and without syrinx, as well as Chiari malformation with hydrocephalus that may or may not be related to the malformation. In one patient, ICP and ICC, as estimated by MR imaging, were changed significantly

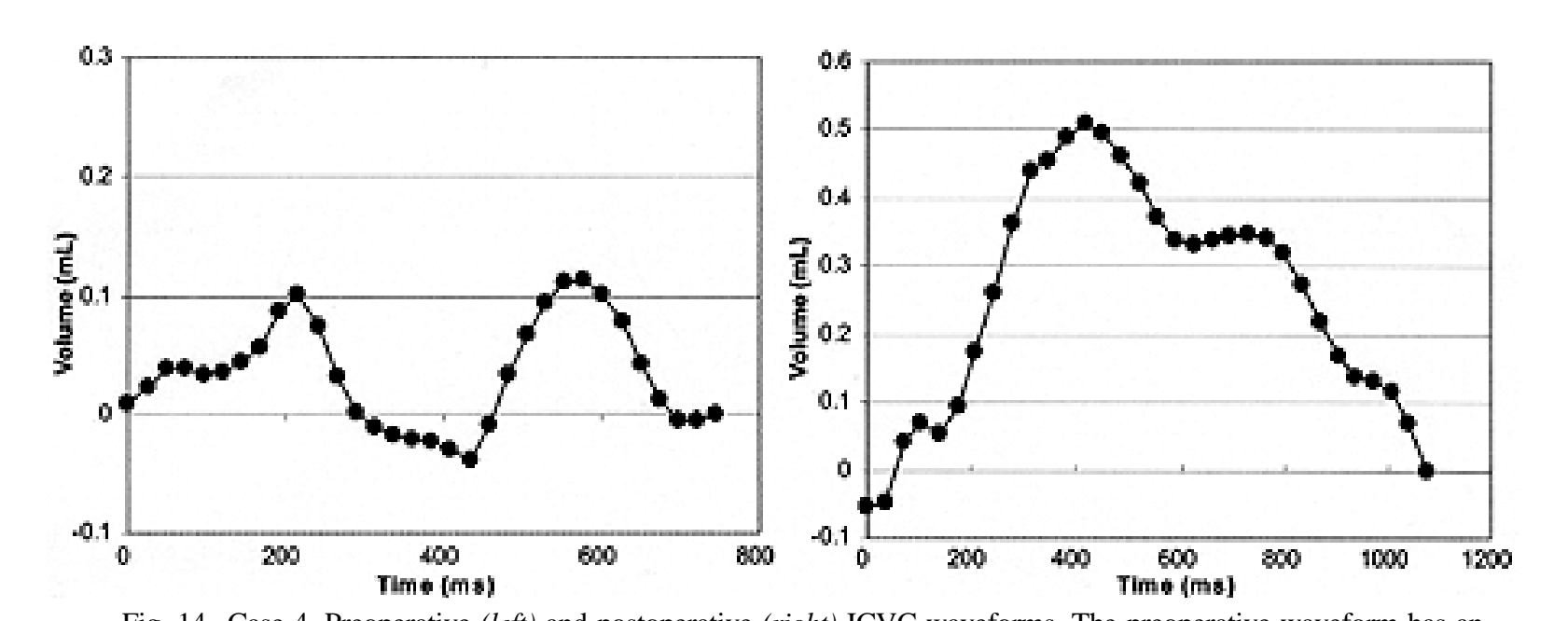

Fig. 14. Case 4. Preoperative (left) and postoperative (right) ICVC waveforms. The preoperative waveform has an abnormal shape of two distinct peaks with markedly decreased amplitude. The preoperative waveform is scaled to emphasize the abnormal shape of ICVC dynamics. The shape of the postoperative waveform represents more normalappearing ICVC dynamics with nearly monotonic decline. 


\section{Blood and CSF measurements in Chiari I malformation}

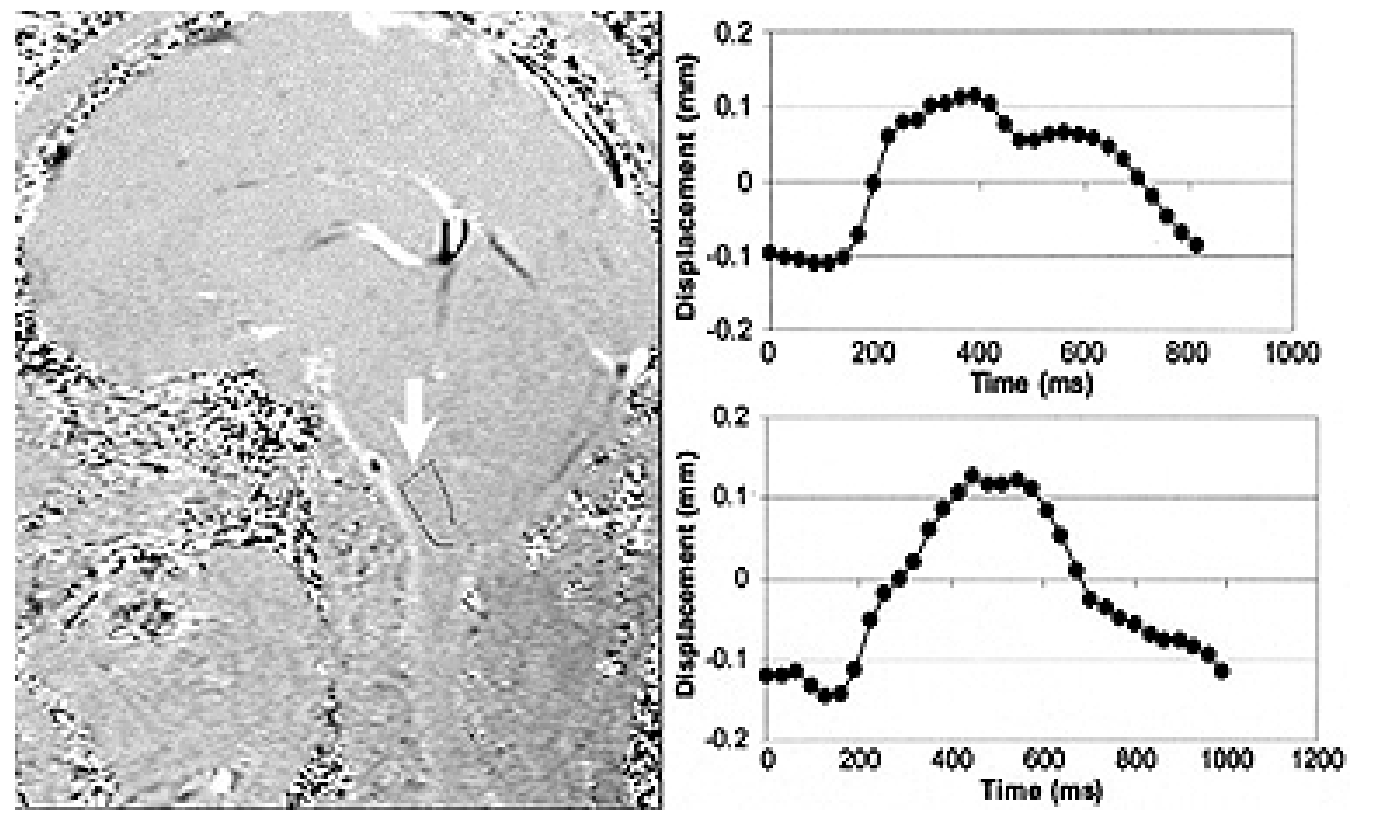

Fig. 15. Case 4. Left: One of the midsagittal velocity-encoded cine phase-contrast MR images acquired during the systolic phase. The brighter intensity indicates velocity in the superoinferior direction. The displacement of the hindbrain region is calculated using the average velocity within the drawn region of interest (white arrow). Preoperative (upper right) and postoperative (lower right) waveforms indicating the hindbrain displacement shown in image at left. Caudal displacement is indicated by positive values. The total hindbrain displacement over the cardiac cycle increased by $17 \%$ (from 0.23 to $0.27 \mathrm{~mm}$ ) after surgery. The relative duration of the caudal displacement is significantly longer in the preoperative state.

by the decompressive intervention. In the other two surgically treated patients no significant change was observed. It is too premature to conclude whether decompressive surgery alleviates the symptoms through a change in the ICC. A larger number of patients with the same disease need to be studied to answer this question.

Regardless of the variable pathological entities present in our patients, there are several common important findings. The first finding is that the ICVC waveform is altered by decompressive surgery. This waveform is derived from the arterial, venous, and CSF flow waveforms. Because blood and CSF flows are coupled ${ }^{2}$ it is important to consider both the hemo- and hydrodynamics when examining the mechanism for the change. The change in the dynamics of the ICVC was associated, to some degree, with a change in CSF flow dynamics. In one patient (Case 4) there was a significant change whereas in the other two surgically treated patients the change was moderate (Case 2) to mild (Case 1). The change in the CSF flow dynamics may be related to the displacement of the hindbrain into the spinal canal during a period of the cardiac cycle, during which, the CSF flow is reduced. We termed this phenomenon a "partial valve" effect. In a previous report, Pujol, et al., ${ }^{14}$ showed a correlation between a displacement index of the midbrain and the symptoms reported by patients. Examining a midsagittal cine phasecontrast MR image, the actual displacement was quantified for Case 4 as demonstrated in Fig. 15 left. In this patient the overall displacement range was a small fraction of $1 \mathrm{~mm}$. This finding provides evidence that the flow modulation may be more significant than the "piston" ef- fect that was proposed in previous studies as an important mechanism for the pathophysiology of the Chiari I malformation. We intend to study hindbrain displacement in healthy individuals and in patients with Chiari I malformation to elucidate further the role of hindbrain displacement during the cardac cycle.

In the two patients without hydrocephalus shorter durations of CSF outflow were shown prior to decompressive surgery. This may be associated with the "partial valve," regulating the CSF flow dynamics and being "closed" for longer portion of the systolic phase (Fig. 15 upper and lower right). Ellenbogen, et al., ${ }^{6}$ showed that CSF flows during shorter portions of the cardiac cycle in patients with Chiari I malformation compared with healthy individuals. This finding together with observations reported here support the role of the partial-valve mechanism in the modulation of the CSF flow as well as the ICVC dynamics.

Another significant finding of this study is that the ICVC waveform is markedly abnormal in patients with headaches. In the patients who suffered headaches prior to surgery (Cases 1 and 4), the ICVC dynamics (Figs. 4 left and 14 left) revealed a double-peak shaped waveform. In these patients, the ICVC dynamics were modified by the decompression to more regular-appearing ICVC waveforms (Figs. 4 left, and 14 left), which were associated with relief of the headaches. In the two patients without headaches (Cases 2 and 3), the ICVC waveforms (Figs. 7 and 11) were more similar to a normal-shaped ICVC waveform. The spontaneous resolution of symptoms in the patient in Case 3 may be explained by remodeling of 
the venous outflow that, overall, yielded a more normalappearing ICVC waveform. In this patient, the buffering of the arterial pulsation may have shifted from the CSF channel to the venous channel.

The preliminary findings presented here may suggest that headaches associated with Chiari I malformations are correlated with abnormal dynamics of the ICVC during the cardiac cycle rather than a change in the overall ICC and ICP. Additional patients need to be studied to validate this hypothesis and to determine whether there is a connection between ICVC and headaches. A possible mechanism may be related to the abnormal stretching of the dura by the irregular increase and decrease in the intradural space represented by the ICVC waveform.

\section{Acknowledgment}

The authors acknowledge the contribution of Balamurali Varadarajalu, Ph.D., from the Department of Radiology at the University of Illinois, for his help in preparing this manuscript.

\section{References}

1. Alperin N, Kadkhodayan Y, Loth F, et al: MRI measurements of intracranial volume change: a phantom study. Proc Intl Soc Mag Reson Med 9:1981, 2001

2. Alperin N, Vikingstad EM, Gomez-Anson B, et al: Hemodynamically independent analysis of cerebrospinal fluid and brain motion observed with dynamic phase constrast MRI. Magn Reson Med 35:741-754, 1996

3. Alperin NJ, Lee SH, Loth F, et al: MR-Intracranial pressure (ICP): a method to measure intracranial elastance and pressure noninvasively by means of MR imaging: baboon and human study. Radiology 217:877-885, 2000

4. Bhadelia RA, Bogdan AR, Wolpert SM, et al: Cerebrospinal fluid flow waveforms: analysis in patients with Chiari I malformation by means of gated phase-contrast MR imaging velocity measurements. Radiology 196:195-202, 1995

5. Bogdanov EI, Ibatullin MM, Mendelevich EG: Spontaneous drainage in syringomyelia: magnetic resonance imaging findings. Neuroradiology 42:676-678, 2000
6. Ellenbogen RG, Armonda RA, Shaw DW, et al: Toward a rational treatment of Chiari I malformation and syringomyelia. Neurosurg Focus 8 (3):Article 6, 2000

7. Ergun R, Akdemir G, Gezici AR, et al: Surgical management of syringomyelia-Chiari complex. Eur Spine J 9:553-558, 2000

8. Heiss JD, Patronas N, DeVroom HL, et al: Elucidating the pathophysiology of syringomyelia. J Neurosurg 91:553-562, 1999

9. Hofmann E, Warmuth-Metz M, Bendszus M, et al: Phase-contrast MR imaging of the cervical CSF and spinal cord: volumetric motion analysis in patients with Chiari I malformation. AJNR 21:151-158, 2000

10. Iwasaki Y, Hida K, Koyanagi I, et al: Reevaluation of syringosubarachnoid shunt for syringomyelia with Chiari malformation. Neurosurgery 46:407-413, 2000

11. Klekamp J, Iaconetta G, Samii M: Spontaneous resolution of Chiari I malformation and syringomyelia: case report and review of the literature. Neurosurgery 48:664-667, 2001

12. Krieger MD, McComb JG, Levy ML: Toward a simpler surgical management of Chiari I malformation in a pediatric population. Pediatr Neurosurg 30:113-121, 1999

13. Munshi I, Frim D, Stine-Reyes R, et al: Effects of posterior fossa decompression with and without duraplasty on Chiari malformation-associated hydromyelia. Neurosurgery 46: 1384-1390, 2000

14. Pujol J, Roig C, Capdevila A, et al: Motion of the cerebellar tonsils in Chiari type I malformation studied by cine phase-contrast MRI. Neurology 45:1746-1753, 1995

15. Sakamoto H, Nishikawa M, Hakuba A, et al: Expansive suboccipital cranioplasty for the treatment of syringomyelia associated with Chiari malformation. Acta Neurochir 141:949-961, 1999

Manuscript received May 16, 2001.

Accepted in final form June 11, 2001.

This work was supported in part by the Ed and Gayle Labuda Charitable Fund of the Vanguard Charitable Endowment Program.

Address reprint requests to: Noam Alperin, Ph.D., Magnetic Resonance Imaging, Center (M/C 711), The University of Illinois at Chicago, 830 South Wood Street, Chicago, Illinois 60612. email: alperin@uic.edu. 